\title{
A ROBUST APPROACH FOR ROAD PAVEMENT DEFECTS DETECTION AND CLASSIFICATION
}

\author{
H. T. Nguyen ${ }^{1}$, thuhuongyb@gmail.com, \\ L. T. Nguyen ${ }^{1}$, thelongit88@gmail.com, \\ D. N. Sidorov ${ }^{1}$, contact.dns@gmail.com. \\ ${ }^{1}$ Irkutsk National Research Technical University, Irkutsk, Russian Federation.
}

\begin{abstract}
The objective of this paper is to propose a robust approach to building a computer vision system to detect and classify pavement defects based on features, such as the contour of feature (chain code histogram, Hu-moment), the shape of an object (length, width, area). In this paper, we present a method to build an automated system to detect and classify the different types of defects such as rupture of the road edge, potholes, subsidence depressions based on image processing techniques and machine learning methods. That system includes the following steps. First step is to detect defect position (ROI) then the defect is described by its features. Finally, each defect is classified these different defect features. In our approach the following algorithms have been using: Markov Random Fields and Graph cuts method for image segmentation, Random Forests algorithm for data classification.

Keywords: feature extraction, defect pavement, defects detection, Markov random fields, Graph cut, Random Forests, Computer vision.
\end{abstract}

\section{Introduction}

Transportation infrastructure development and maintenance is essential part of economy. The good quality roads are a necessity for nearly every human activity. For effective management of the road networks, one needs accurate and up to date information about road pavement defects. Thousands of kilometers of road pavement need to be inspected each year. Earlier, road defects information was obtained manually by human inspectors. But such manual methods are very slow and uncomfortable for inspectors and road users. In the last years, several automated inspecting techniques were implemented. Many of these state-of-the-art technologies involve machine vision and machine learning method. The objective of this article is to contribute to this field. Defect detection problem becomes especially difficult for noisy surfaces. There are many types of texture can be encountered on the road. In addition, texture depends on current zones of the image due to different regions. Moreover, texture can have big aggregate size. Due to these reasons, it can be difficult to distinguish crack and part with extraordinary texture. Road pavement defects exist in many forms such as rupture of the road edge, cracks (grid cracking, large crack), potholes, subsidence depressions are described in figure 1.

Each form of road pavement has got certain features, which are not the same, help us to distinguish them. If we only consider the simple features such as shape descriptors, region descriptors (length, width, area) the data is unclear and difficult to apply defects road pavement recognition. An image can be considered as a mosaic of different texture regions, and the image features associated with these regions can be used for recognition. The purpose of this paper is to study the use a combination of different types of features, in particular, textural. The article is organized as follows. First, we provide a brief overview of related work. Then we describe defect pavement detection method and improve a quality of 


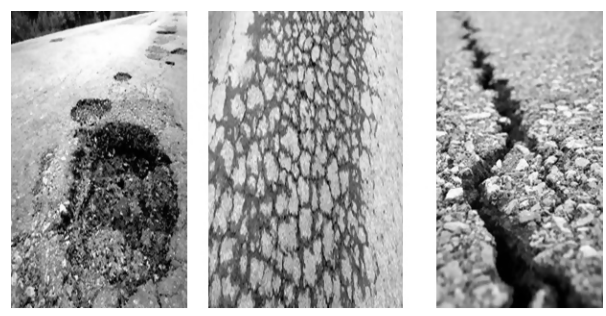

Fig. 1. Defects type of pavement road

image segmentation by Markov random fields and Graph cuts method. Finally, we present data classification based on Random Forests algorithm and conclusions.

\section{Related Work}

Advances in computer technology in the field of digital image processing allow us to use it for analyzing pavement road. The analysis of pavement road image showing defects pavement detection due to improving the accuracy and the quality of defects pavement classification. In defects pavement detection task for can be classified in two group. There are model-based, which follow a top-down approach, only the features included in the model are found. Therefore, it is difficult to build a model for new roads. The second group is features-based, which follow a bottom-up approach. All the features that are in the image are subject to be found, but noise can generate false detections. Several researchers have considered the use of such texture features for pattern retrieval [11]. In [6] authors presented a method for automated pothole detection in asphalt pavement images using histogram shape-based thresholding in order to segment an image into a defect and nondefect regions. In [5] Wang S, Tang W introduced a method to segment the pavement crack image by finding the local optimal threshold value based on the crack density distribution. A good starting point can be found in [3] which reviews the techniques applied for the development of automatic pavement distress detection and classification system. They also propose a novel approach according to the following major steps: region based on image enhancement, to correct non-uniform background illumination and a skeleton analysis algorithm to classify pavement surface distress types. In [2] authors proposed a presented a neural network based technique for the classification of segments of road images into cracks and normal images. The density and histogram features are extracted. The features are passed to a neural network for the classification of images into images with and without cracks. Once images are classified into cracks and non-cracks, they are passed to another neural network for the classification of a crack type after segmentation. Graphical model widely used for segmentation. In [9] authors proposed two novel methods for road lane marking and road surface artifacts detection. These algorithms are developed for videobased road registration and monitoring system, which is car-mounted complex for data gathering and analysis of road surface. Detection is performed on rectified images of the road surface, constructed from video sequences from driving vehicle. A new method of road lane marking detection is based on machine-learning approach. The algorithm applies to segmentation method to images and then classify the regions using classifier cascades. In [8] Lempert, Sidorov and Zhukov presented an approach to the problem of prioritization work on repairing the pavement with limited resources, which is to use a combination of 
methods for identification and classification of defects on the basis of statistical analysis and machine learning (Random Forests) with original methods for solving the infinitedimensional optimization (optical-geometrical analogy).

\section{System of Computer Vision for Defects Pavement Detection and Classification}

In this section, we present the issues related to computer vision system for detecting and classifying defects pavement. Including parts: Data collection, preprocessing image based on morphological operations, noise filtering, smoothing to improve image quality. Next features extraction, defect pavement segmentation based on two methods: Graph cuts and Markov Random Fields, Finally we present defects pavement classification by Random Forests.

\subsection{Defects Road Pavement Data Collection}

Input data of the system are actual defects road pavement on local roads [4] collected by authors of this article. Apart from dataset [4] we also used images provided by Center for Telecommunications and Multimedia, INESC TEC, Portugal [10]. The collection is supported by video recording devices and camera (Canon D100 16 megapixel). Images are captured in conventional daylight condition. The distance from the lens to the surface road is in the range from 1 meter to 1.2 meters. Then the photos were collected and processed at one resolution $(256 * 256)$ pixels and $(500 * 500)$ pixels. The training set consists of 500 images (200 of class 'defect' and 300 of class 'non-defect'). In 200 images of class, defect included 150 images (50 Block images, 50 Longitudinal images, 50 Pothole images) for training process and 50 images for the testing process. In 300 images of class, non-defect included 200 for training process and 100 images for the testing process. Imbalanced data follows the idea of cost-sensitive learning makes Random Forests more suitable for learning. Class weights are an essential tuning parameter to achieve desired performance. In the tree induction procedure, class weights are used to weight the Gini criterion for finding splits. In the terminal nodes of each tree, class weights are again taken into consideration. We introduce the concept: True Positive $-T P$ is classified correctly as positive, True Negative $-T N$ is classified correctly as negative, False Positive $-F P$ is classified wrongly as positive, False Negative $-F N$ is classified wrongly as negative. For Random Forests algorithm, there is always a trade-off between true positive rate and true negative rate and the same applies for recall and precision. True nagative rate $=T N / T N+F P$, True Positive rate $=T P / T P+F N$, Precision $=T P / T P+F P$.

\section{Table 1}

Best Model for Classification Depends upon the Precision, True Positive Rate, False Positive Rate

\begin{tabular}{|c|c|c|c|}
\hline Class & True Positive & False Positive & Precision \\
\hline Potholes & 0.903 & 0.556 & 0.843 \\
\hline Block crack & 0.80 & 0.726 & 0.880 \\
\hline Longitudinal cracks & 0.947 & 0.230 & 0.926 \\
\hline
\end{tabular}




\subsection{Preprocessing Image Based on Morphological Operations}

We propose to preprocess images before the feature extraction. First, we apply noise filtering using Gaussian filter and convert to grayscale images. On the next step, we perform image segmentation. We propose to divide the image into separate regions. Then we separate pixel defects to detect a connected region. We use the morphological method to detect pixels corresponding to defects and to remove small regions which are considered as noise. Mathematical morphology analyses the geometrical structure of a set by probing and transforming its microstructure with different predefined elementary sets [7].

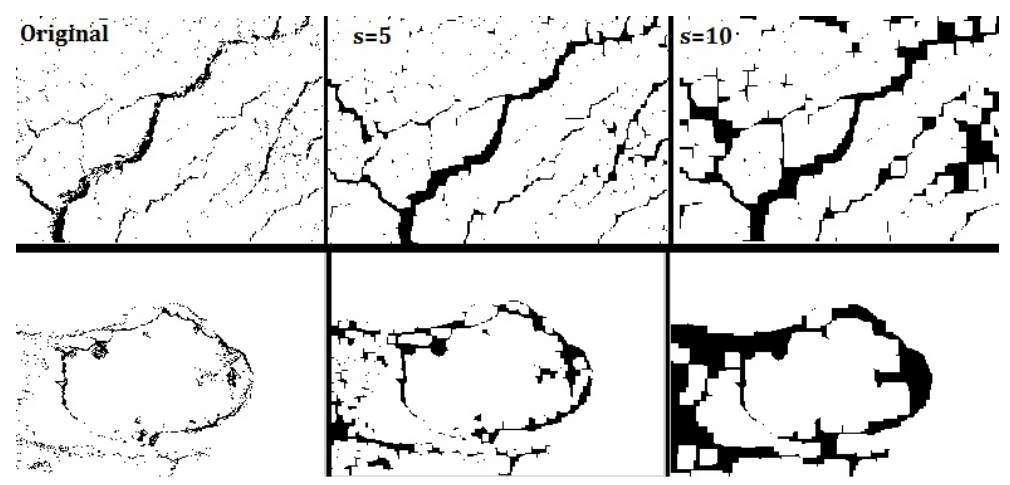

Fig. 2. Defects road pavement after used dilation and erosion morphological operations.

Figure 2 shown results of using dilation and erosion morphological operations to extract defects road pavement position in images. The result of the process of finding regions of pavement defects have shown that we can change the size of the structure element $(s=5$, $s=10, \ldots)$ to identify values of each pixel in image and gave to us perfectly extract defect regions.

\subsection{Features Extraction}

In this paper, we consider the following defects.

Block crack. Interconnected cracks forming a series of blocks approximately rectangular in shape, commonly distributed over the full pavement. Attributes of block crack defect are: Predominant crack width $(\mathrm{mm})$, predominant cell width $(\mathrm{mm})$, area affected $\left(\mathrm{m}^{2}\right)$.

Longitudinal Cracks. Unconnected crack running longitudinally along the pavement. Attributes of Longitudinal Cracks defect are Crack width $(\mathrm{mm})$, Crack length $(\mathrm{m})$, Crack spacing $(\mathrm{mm})$, Area affected $\left(\mathrm{m}^{2}\right)$.

Potholes. Irregularly shaped holes of various sizes in the pavement. Attributes of Potholes defect are depth of potholes $(\mathrm{mm})$ and area of pothole $\left(\mathrm{m}^{2}\right)$.

For the analysis of the attributes of each defect, we selected the following features:

Hu-moments: The most notable are Hu-Moments which can be used to describe, characterize, and quantify the shape of an object in an image. Hu-Moments are normally extracted from the shape of an object in an image. By describing the shape of an object, we are able to extract a shape feature vector (i.e. a list of numbers) to represent the shape of the object. 
Chain code histogram: The chain code histogram $(\mathrm{CCH})$ is meant to group together objects that look similar to a human observer. It is not meant for exact detection and classification tasks. The $\mathrm{CCH}$ is calculated from the chain code presentation of a contour.

The Freeman chain code is a compact way to represent a contour of an object. The chain code is an ordered sequence of $n \operatorname{links}\left\{c_{i}, i=1,2, . ., n\right\}$, where $c_{i}$ is a vector connecting neighboring contour pixels. The directions of $c_{i}$ are coded with integer values $k=0,1, \ldots, K-1$ in a counterclockwise sense starting from the direction of the positive $x$-axis. The number of directions $K$ takes integer values $2^{M+1}$ where $M$ is a positive integer. The chain codes where $K>8$ are called generalized chain codes. The calculation of the chain code histogram is fast and simple. The $\mathrm{CCH}$ is a discrete function:

$$
p(k)=n_{k} / n, k=0,1, \ldots, K-1,
$$

where $n_{k}$ is the number of chain code values $k$ in a chain code, and $n$ is the number of links in a chain code. Beside we consider also size of defect region (width and length, area) and histogram of image.

\subsection{Defect Pavement Segmentation Based on Graph Cuts Method and Markov Random Fields Method}

Defect pavement detection, where image region are labeled as containing defect pixels or not, and defect pavement type classification, where 'Block crack', 'Longitudinal Cracks', 'Potholes' labels are assigned to each detected defect pavement. For defect pavement detection, an initial setup is required where the operator selects images used to determine an optimum set of detection parameters accounting for pixel-by-pixel gray scale variation as related to defect pavement contrast, brightness, and surface conditions. An example is a digital defect map as shown in Fig. 3 demonstrates defect map corresponding to images shown on Fig. 4.

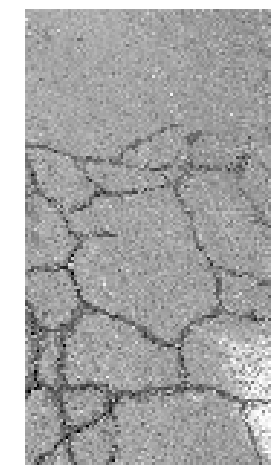

Fig. 3. Digital pavement image

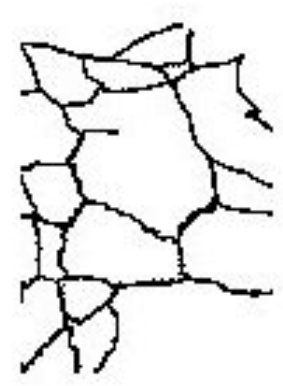

Fig. 4. Defect pavement map

To improve quality of image segmentation (Fig. 5), with a Markov random fields [12] is used. These segments are called 'sites' and have a predefined orientation of $0,45,90$ or 135 degrees. The separation between both cases is done with parameter $k \in(0,1)$. Our goal will be to segment an image by constructing a graph such that the minimal cut of this graph will cut all the edges connecting the pixels of different objects with each other. 


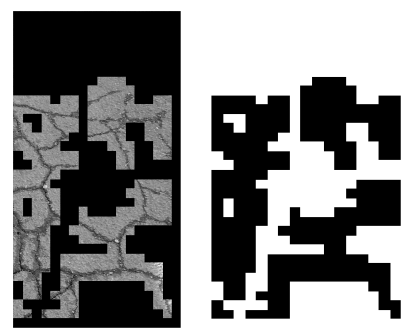

Fig. 5. Defect pavement image segmentation.

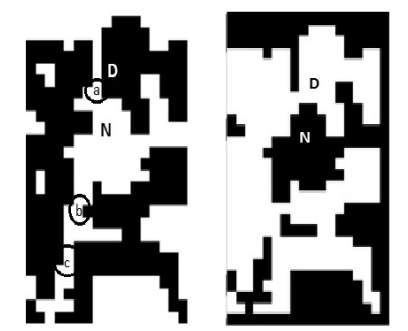

Fig. 6. Use Graph cut to improve image segmentation.

Algorithm. Steps of Graph cut method

1. Start with an arbitrary labeling $f$

2. Set success $:=0$

3. for each pair of labels $D, N \subset L$ do

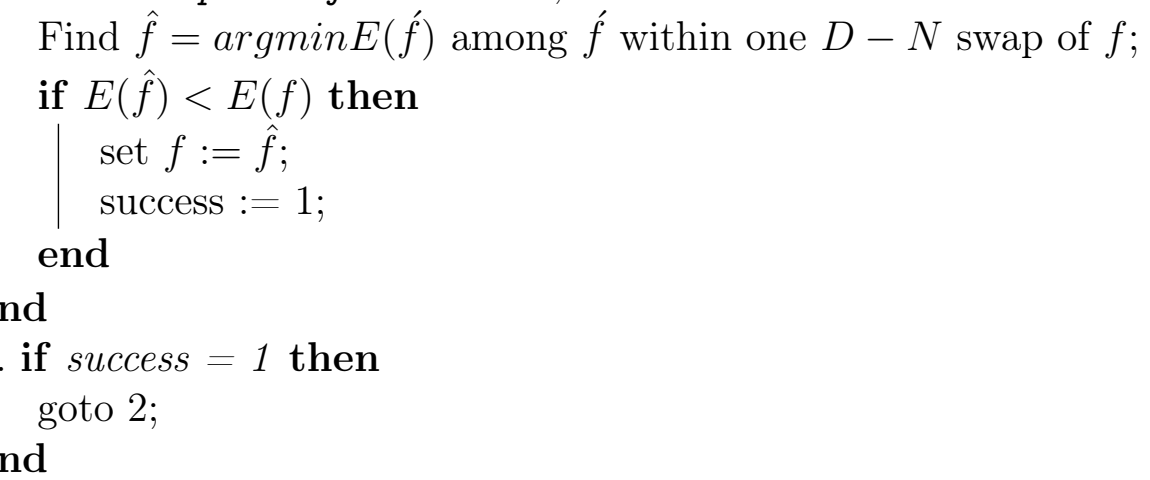

5. Return $f$

We applied efficient graph based method to find the optimal D (Defect)-N (Not defect) as shown in Fig. 6 swap or D - expansion given a labeling $f$. We use graph cuts to efficiently find $\hat{f}[14],[13]$. Let us briefly outline the approach we used.

Let $G=\langle V, E\rangle$ be a weighted graph with two distinguished verticals called the terminals. A cut $C \in E$ is a set of edges such that the terminals are separated in the induced graph $G(C)=\langle V, E-C\rangle$. In addition, no proper subset of $\mathrm{C}$ separates the terminals in $G(C)$. The cost of the cut $C$, denoted $|C|$, equals the sum of its edge weights. A graph-based approach makes use of efficient solutions of the maxflow/mincut problem between source and sink nodes in directed graphs. To take advantage of this we generate an s-t-graph as follows: The set of nodes is equal to the set of pixels in the image. Every pixel is connected with its d-neighborhood $(d=4 ; 8)$. The minimum cut problem is to find the cheapest cut among all cuts separating the terminals. Minimum cuts can be efficiently found by 
standard combinatorial algorithms with different low-order polynomial complexities [16]. Our experimental results have been obtained using a new max-flow algorithm that has the best speed on our graphs over many modern algorithms. The method Graph Cuts finds the optimal solution to a binary problem. However, when each pixel can be assigned many labels, finding the solution can be computationally expensive. For the following type of energy, series of graph cuts can be used to find a convenient local minimum. Such that follows:

$$
E(f)=\sum_{p \in P} D_{p}\left(i_{p}, f_{p}\right)+\sum_{p, q \in N} V_{p, q}\left(f_{p}, f_{q}\right)
$$

where $N \subset P \times P$ is a set of neighborhood pixels. $D_{p}\left(f_{p}\right)$ is a function derived from the observed data that measures the cost of assigning the label $f_{p}$ to the pixel $p$. $V_{p, q}\left(f_{p}, f_{q}\right)$ measures the cost of assigning the labels $f_{p}, f_{q}$ to the adjacent pixels $p, q$ and is used to impose spatial smoothness. Energy functions of the form (1) can be justified on Bayesian grounds using the well-known Markov Random Fields (MRF) formulation [17]. The running time is nearly linear in practice. Some results of segmentation of classes defect road pavement are shown in Fig. 7.

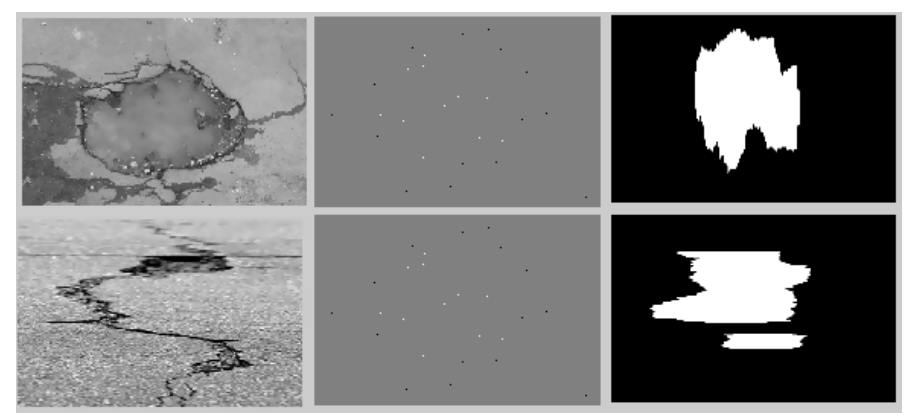

Fig. 7. Results of segmentation of classes defect pavement.

\subsection{Defect on Road Pavement Classification by Random Forests Method}

This section describes the classification based on unsupervised learning method approach (Fig. 8) Random Forests [15]. A Random Forests algorithm takes the decision tree concept further by producing a large number of decision trees. The approach first takes a random sample of the data and identifies a key set of features to grow each decision tree. These decision trees then have their Out-Of-Bag error determined (error rate of the model) and then the collection of decision trees are compared to find the joint set of variables that produce the strongest classification model. Let us briefly outline the basic idea of a Random Forests algorithm below:

- At each tree split, a random sample of $m$ features is drawn, and only those $m$ features are considered for splitting. Typically or, where $\mathrm{p}$ is the number of features.

- For each tree grown on a bootstrap sample, the error rate for observations left out of the bootstrap sample is monitored. This is called the 'out-of-bag' error rate.

The whole database of training images features compose a pattern vector feature $x$, representing a sample of the random variable $X$, taking values on a sample space $X$. For each element $x_{i}$ of pattern vector $x$, one possible class $y_{i}$ is assigned, where $Y$ is the class 


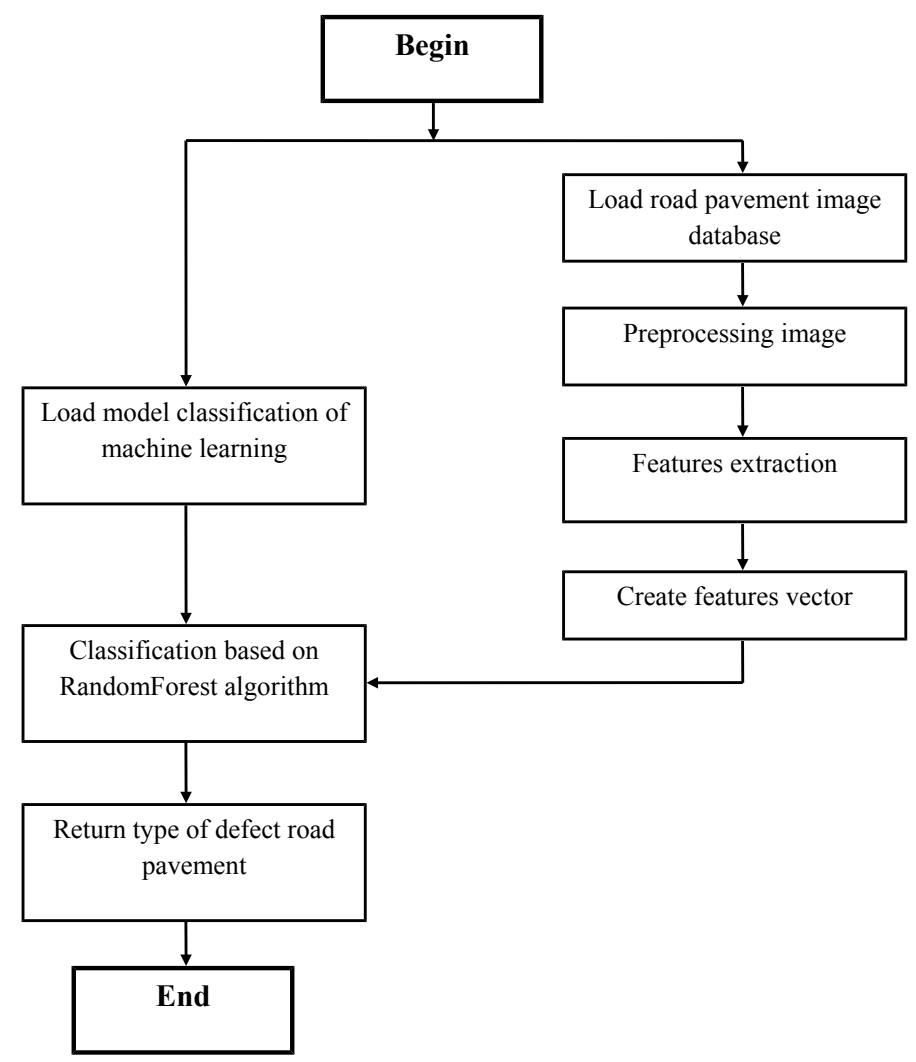

Fig. 8. Defect of pavement classification data flow diagram.

set, $y_{i} \in Y$. The training set is:

$$
T=\left\{\left(x_{1}, y_{1}\right) \ldots\left(x_{n}, y_{n}\right): x_{i} \in \mathbb{R}^{2} ; y_{i} \in\left\{c_{1}, c_{2} \ldots, c_{n}\right\}\right\}
$$

Here $n$ is the number of points of the pattern vector $x$.

The Random Forests classifier was built using the package Random Forests 4.5-16 for the $\mathrm{R}$ statistical environment [18] to classify feature vectors as defect or non-defect. The classifier was trained on pavements road dataset using Chain code histogram $\mathrm{CCH}, \mathrm{Hu}$ moments, size of defect for each variant. We also used Boosting (GBTs) to classify this dataset and to compare results from two classification methods. The main difference between these two algorithms is the order in which each component tree is trained. The classifier was built using the parameters $n t r e e=(50,100)$ and $m t r y=2$ and depth $=(2,5)$. Table 2 shows the effect of increasing the number of trees in the ensemble. For both, increasing trees require more time to learn but also provide better results in terms of Mean Squared Error (MSE) is calculated as follows:

$$
M S E=\frac{1}{n} \sum_{i=1}^{n}\left(f\left(x_{i}\right)-y_{i}\right)^{2} .
$$

Here $n$ is the number of test examples, $f\left(x_{i}\right)$ the classifier's probabilistic output on $x_{i}$ and $y_{i}$ are actual labels.

Random Forests are fast to train, but they often require deep trees. Random Forests do not overfit as easily, but algorithm's test error plateaus. 
Table 2

Results of classification algorithms: Random Forests and Boosting(GBTs)

\begin{tabular}{|c|c|c|c|c|}
\hline & \multicolumn{2}{|c|}{ Random Forests } & \multicolumn{2}{c|}{ Boosting(GBTs) } \\
\cline { 2 - 5 } & Trees:50 & Trees:100 & Trees:50 & Trees:100 \\
& Depth:2 & Depth:5 & Depth:2 & Depth:5 \\
\hline Training time(sec) & 150 & 257 & 140 & 278 \\
Correct rate(\%) & 80.5 & 93.29 & 88.57 & 91.45 \\
MSE & 0.393 & 0.366 & 0.3 & 0.516 \\
\hline
\end{tabular}

Our experiments show that more trees are always better with diminishing returns. Deeper trees are almost always better subject to requiring more trees for similar performance. The above two points are directly a result of the bias-variance trade off. Deeper trees reduces the bias; more trees reduces the variance. There are several ways to control how deep our trees are (limit the maximum depth, limit the number of nodes, limit the number of objects required to split, stop splitting if the split does not sufficiently improve the fit, ...). Most of the time, it is recommended to prune (limit the depth of) the trees if we are dealing with noisy data. Finally, we can use our fully developed trees to compute performance of shorter trees as these are a 'subset' of the fully developed ones.

\section{Conclusions}

In this paper, we propose robust approach to construct a computer vision system for the automatic detection and classification of defects road pavement. By using image processing techniques combined with unsupervised learning methods to build effective detection and classification system. we have presented a defining defect pavement contour based on morphological operator which allows us to extract defect position. A simple boosting method is used to train the classifier and the two sets (one for each road) make it possible to achieve results which demonstrate the robustness of the implemented method and algorithm for pavement crack detection based on Markov Random Fields. We also propose to use to Graph cut method, which improves quality of image segmentation and can detection part of pavement defect - nondefect. The classification algorithm - Random Forests was able to correctly classify all the images contained in the three classes.

\section{References}

1. Charles A.B., Sauer K., Saquib S. Markov Random Fields and Stochastic Image Models. IEEE International Conference on Image Processing, October 23-26, 1995. Washington, D.C., 1995.

2. Bray J., Verma B., Li X., He W. A Neural Network Based Technique for Automatic Classification of Road Cracks. Proceeding of the International Joint Conference on Neural Networks, July 16-21, 2006. Vancouver, BC, Canada, 2006, pp. 907-912.

3. Chen H., Miyojim M. Automatic Pavement Distress Detection System. Information Sciences, 1998, vol. 108, issues 1-4, pp. 219-240. doi: 10.1016/S0020-0255(97)10062-7 
4. Pavement Defects Dataset, available at: https://yadi.sk/d/GEIaJHYOspQbR (accessed on 27 June 2016).

5. Wang S., Tang W. Pavement Crack Segmentation Algorithm Based on Local Optimal Threshold of Cracks Density Distribution. Proceedings of the Advanced Intelligent Computing - 7th International Conference ICIC, August 11-14, 2011. Zhengzhou, China, Springer, 2011, pp. 298-302. doi: 10.1007/978-3-642-24728-6_40

6. Koch C., Brilakis I. Pothole Detection in Asphalt Pavement Images. Advanced Engineering Informatics, 2011, vol. 25, no. 3, pp. 507-515.

7. Toet A. A Morphological Pyramidal Image Decomposition. Pattern Recog. Lett., 1989, vol. 9, pp. 255-261.

8. Lempert A.A., Sidorov D.N., Zhukov A. V. An Approach to Optimization of Repair of Roads in the Conditions of Limited Financing. VII International Symposium "Generalized Statements and Solutions of Control Problems" (GSSCP-2014), Gelendzhik, September 26-30, 2014. Moscow, Izdatel'stvo fiziko-matematicheskoj literatury Publ., 2014, pp. 114-118. (in Russian).

9. Sudakov S., Semashko A., Barinova O., Konushin A. Kinshakov V., Krylov A. Detection of Road Lane Marking and Artifacts of Road Surface. Graphicon 2008 Proceedings, 2008, pp. 206-212. (in Russian).

10. Fernandes K., Ciobanu L. Pavement Pathologies Classification Using Graph-Based Features. IEEE International Conference on Image Processing (ICIP), October 2730, 2014. Paris, France, IEEE, 2014. doi: 10.1109/ICIP.2014.7025159

11. Manjunath B.S., Wei-Ying Ma. Texture Features for Browsing and Retrieval of Image Data. IEEE Transactions on Pattern Analysis and Machine Intelligence, August 1996, 1996, vol. 18, no. 8, pp. 837-842.

12. Delagnes P., Dominique B. A Markov Random Fields for Rectilinear Structure Extraction in Pavement Distress Image Analysis. International Conference on Image Processing, January 1995, 1995, vol. 1, pp. 446-449. doi: 10.1109/ICIP.1995.529742.

13. Kolmogorov V., Zabih R. Computing Visual Correspondence with Occlusions via Graph Cuts. Eighth IEEE International Conference on Computer Vision, February 2001, 2001, vol. 2, pp. 508-515. doi: 10.1109/ICCV.2001.937668

14. Boykov Y., Veksler O., Ramin Z. Fast Approximate Energy Minimization Via Graph Cuts. IEEE Transactions on Pattern Analysis and Machine Intelligence, December 2001, 2001, vol. 23, no. 11, pp. 1222-1239. doi: 10.1109/34.969114

15. Breiman L. Random Forests. Machine Learning, 2001, vol. 45, issue 1, pp. 5-32. doi: 10.1023/A:1010933404324

16. Ravindra K. A., Magnanti T.L., Orlin J.B. Network Flows: Theory, Algorithms and Applications. Prentice Hall, 1993. doi: 10.2307/2583863 
17. Geman S., Geman D. Stochastic Relaxation, Gibbs Distributions, and the Bayesian Restoration of Images. IEEE Transactions on Pattern Analysis and Machine Intelligence, November 1984, 1984, vol. 6, no. 6, pp. 721-741. doi:10.1109/TPAMI.1984.4767596

18. $R$ language packet, available at: https://www.r-project.org/. (accessed on 03 September 2015).

Nguyen Thu Huong, Postgraduate, Irkutsk National Research Technical University (Irkutsk, Russian Federation), thuhuongyb@gmail.com

Nguyen The Long, Postgraduate, Irkutsk National Research Technical University (Irkutsk, Russian Federation), thelongit88@gmail.com

Denis N. Sidorov, Doctor of Physico-Mathematical Sciences, Full Professor, Department of Computer Science, Irkutsk national research technical university, (Irkutsk, Russian Federation), contact.dns@gmail.com

Received June 30, 2016

УДК 51.74

DOI: $10.14529 /$ jcem160305

\title{
УСТОЙЧИВЫЙ ПОДХОД ДЛЯ РЕШЕНИЯ ЗАДАЧИ ОБНАРУЖЕНИЯ И КЛАССИФИКАЦИИ ДЕФЕКТОВ ДОРОЖНОГО ПОКРЫТИЯ
}

\author{
Т.Х. Нгуен, Т.Л. Нгуен, Д.Н. Сидоров
}

\begin{abstract}
Целью данной работы является разработка устойчивого подхода к построению системы компьютерного зрения для обнаружения и классификации дефектов дорожного покрытия на основе признаков. В этой статье мы представляем метод построения автоматизированной системы для обнаружения и классификации различных типов дефектов, таких как разрыв края дороги, выбоины, просадочных депрессий на основе методов обработки изображений и алгоритмов машинного обучения. Система включает в себя следующие шаги. На первом этапе, определяется положение дефекта (ROI). На втором этапе, дефект описывается его признаками. Последний шаг заключается в классификации каждого дефекта, используя эти различные признаки дефектов. В нашем подходе были использованы следующие алгоритмы: Марковские случайные поля и метод разреза графов для сегментации изображений, алгоритм случайных лесов для классификации данных.

Ключевые слова: извлечение признаков, дефект дорожного покрытия, обнаружение дефектов, Марковские случайные поля, разрез графов, компъютерное зрение.
\end{abstract}




\section{Литература}

1. Charles, A.B. Markov Random Fields and Stochastic Image Models / A.B. Charles, K. Sauer, S. Saquib // IEEE International Conference on Image Processing, October 23-26, 1995. - Washington D.C., 1995.

2. Bray, J. A Neural Network Based Technique for Automatic Classification of Road Cracks / J. Bray, B. Verma, X. Li, W. He // Proceeding of the International Joint Conference on Neural Networks, July 16-21, 2006. - Vancouver, BC, Canada, 2006. P. 907-912.

3. Chen, H. Automatic Pavement Distress Detection System / H. Chen, M. Miyojim // Information Sciences. - 1998. - V. 108, is. 1-4. - P. 219-240.

4. Pavement Defects Dataset, доступ: https://yadi.sk/d/GEIaJHYOspQbR (запрос 27 июня 2016).

5. Wang, S. Pavement Crack Segmentation Algorithm Based on Local Optimal Threshold of Cracks Density Distribution / S. Wang, W. Tang // Proceedings of the Advanced Intelligent Computing - 7th International Conference ICIC, August 11-14, 2011. Zhengzhou, China: Springer, 2011. - P. 298-302.

6. Koch, C. Pothole Detection in Asphalt Pavement Images / C. Koch, I. Brilakis // Advanced Engineering Informatics. - 2011. - V. 25, №. 3. - P. 507-515.

7. Toet, A. A Morphological Pyramidal Image Decomposition / A. Toet // Pattern Recog. Lett. - 1989. - V. 9. - P. 255-261.

8. Лемперт, А.А. Об одном подходе к оптимизации ремонта автомобильных дорог в условиях ограниченного финансирования / А.А. Лемперт, Д.Н. Сидоров, А.В. Жуков // VII Международный симпозиум «Обобщенные постановки и решения задач управления», Геленджик, Дивноморское, 26-30 сентября 2014. - М.: Издательство физико-математической литературы, 2014. - С. 114-118.

9. Судаков, С. Алгоритмы детектирования разметки и дефектов дорожного покрытия / С. Судаков, А. Семашко, О. Баринова, А. Конушин, В. Киншаков, А. Крылов // Graphicon 2008 Proceedings. - 2008. - С. 206-212.

10. Fernandes, K. Pavement Pathologies Classification Using Graph-Based Features K. Fernandes, L. Ciobanu // IEEE International Conference on Image Processing (ICIP), October 27-30, 2014. - Paris, France: IEEE. - 2014.

11. Manjunath, B.S. Texture Features for Browsing and Retrieval of Image Data B.S. Manjunath, Ma Wei-Ying // IEEE Transactions on Pattern Analysis and Machine Intelligence, August 1996. - 1996. - V. 18, №. 8. - P. 837-842.

12. Delagnes, P. A Markov Random Fields for Rectilinear Structure Extraction in Pavement Distress Image Analysis / P. Delagnes, B. Dominique // International Conference on Image Processing, January 1995. - 1995. - V. 1. - P. 446-449. 
13. Kolmogorov, V. Computing Visual Correspondence with Occlusions via Graph Cuts / V. Kolmogorov, R. Zabih // Eighth IEEE International Conference on Computer Vision, February 2001. - 2001. - V. 2. - P. 508-515.

14. Boykov, Y. Fast Approximate Energy Minimization Via Graph Cuts / Y. Boykov, O. Veksler, Z. Ramin // IEEE Transactions on Pattern Analysis and Machine Intelligence, December 2001. - 2001. - V. 23, №. 11. - P. 1222-1239.

15. Breiman, L. Random Forests / L. Breiman // Machine Learning. - 2001. - V. 45, is. 1. - P. 5-32.

16. Ravindra, K.A. Network Flows: Theory, Algorithms and Applications K.A. Ravindra, T.L. Magnanti, J.B. Orlin. - Prentice Hall, 1993.

17. Geman, S. Stochastic Relaxation, Gibbs Distributions, and the Bayesian Restoration of Images / S. Geman, D. Geman // IEEE Transactions on Pattern Analysis and Machine Intelligence, November 1984. - 1984. - V. 6, №. 6. - P. 721-741.

18. R language packet, доступ: https://www.r-project.org/. (запрос 03 сентября 2015).

Нгуен Тху Хыонг, аспирант, Иркутский начиональный исследовательский технический университет (г. Иркутск, Российская Федерачия), thuhuоngyb@gmail.com

Нгуен Тхе Лонг, аспирант, Иркутский национальный исследовательский технический университет (е. Иркутск, Российская Федеращия), thelongit88@gmail.com

Сидоров Денис Николаевич, доктор физико-математических наук, профессор, кафедра вычислительной техники, Иркутский начиональный исследовательский технический университет (2. Иркутск, Российская Федерачия), contact.dns@gmail.com

Поступила в редакцию 30 июня 2016 г. 